\title{
How would Widespread Community Transmission of Covid-19 in Sri Lanka look like? A Population- based Prediction
}

N.W.A.N.Y.Wijesekara ( $\square$ novil.wijesekara@gmail.com )

Disaster Preparedness and Response Division, Ministry of Health, Sri Lanka https://orcid.org/00000002-0391-6220

\section{H.D.B. Herath}

Disaster Preparedness and Response Division, Ministry of Health, Sri Lanka

\section{K.A.L.C. Kodithuwakku}

Disaster Preparedness and Response Division, Ministry of Health, Sri Lanka

\section{H.M.M.N.K.Herath}

Post Graduate Institute of Medicine, University of Colombo.

\section{B.A.M.P. Bulathsinhala}

Post Graduate Institute of Medicine, University of Colombo.

\section{C.C. Magedaragamage}

Post Graduate Institute of Medicine, University of Colombo.

\section{Research Article}

Keywords: Covid-19, Community Transmission, SIR Model, CHIME, Outbreak, Prediction

Posted Date: November 3rd, 2020

DOl: https://doi.org/10.21203/rs.3.rs-101037/v1

License: (c) (1) This work is licensed under a Creative Commons Attribution 4.0 International License. Read Full License 


\section{Abstract}

Covid-19 is a viral disease which has briskly invaded the globe, Sri Lanka being no exception. If community transmission of Covid-19 occurs, it will have serious demands on Sri Lanka's free health care system. Objective of this study was to simulate the widespread community transmission of Covid-19 in Sri Lanka. We used the Susceptibility, Infected and Removed (SIR) model through the Penn State University CHIME Model incorporated to ArcGIS Pro. We simulated introduction of one case of Covid-19 to each of the 26 health districts and ran the model for 365 days. During simulated scenario, the number patients requiring admissions, ICU care and mechanical ventilation will peak at 1942, 583 and 388 per day respectively around 213 days from the onset of widespread community transmission. The cumulative number of cases needing admission, ICU care and ventilation will be 245,916, 73,775 and 49,183 after 365 days. Colombo and Gampaha districts will report the highest number of daily total numbers of hospitalized cases, each which will be over 1680. Health authorities must be ready for the worst-case scenarios of the Covid-19 outbreak to sustain public health response to reduce morbidity and mortality.

\section{Highlights}

\section{What we already know}

- Prediction models could be used for operational readiness of the health sector in anticipation of the Covid-19 outbreak.

- Susceptibility, Infected and Removed (SIR) model, is one of the simplest compartmental epidemiological models used in outbreak predictions, assuming that during widespread community transmission, the population would be fairly homogeneous in relation to disease dynamics.

- Sri Lanka has observed and managed several clusters of Covid-19.

\section{What this article adds}

- Under the simulation scenario, the number patients requiring admissions, ICU care and mechanical ventilation in Sri Lanka will peak at 1942, 583 and 388 per day respectively around 213 days from the onset of widespread community transmission.

- The model shows that the cumulative number of cases needing admission, ICU care and ventilation to be $245,916,73,775$ and 49,183 after 365 days from the onset of widespread community transmission.

- During a possible community transmission and beyond, Colombo and Gampaha districts will report the highest number of daily total numbers of hospitalized cases, of which each will be over 1680 . 


\section{Introduction}

Covid-19 is a viral disease that has spread across many countries in seven months of its existence on earth. As of 29.10.2020, globally there have been a total of 44,351,506 cases and 1,171,255 deaths (World Health Organization 2020b). In the meantime, Sri Lanka has reported 9791 cases and 19 deaths by the same date (Epidemiological Unit 2020). The world Health Organization recommends a comprehensive package of measures for countries to prepare when there are no cases, sporadic cases, clusters of cases, community transmission, or country-wide transmission (World Health Organization 2020a). Although often misinterpreted, WHO identifies community transmission as "large outbreaks of local transmission defined through an assessment of factors including, but not limited to large number of cases not linkable to transmission chains, large number of cases from sentinel lab surveillance, and multiple unrelated clusters in several areas of country/territory/area".

Determining if a country is in community transmission, or country-wide transmission could be quite controversial, as it has been evident from India (Singhania 2020). The decision might carry not only far reaching public health consequences, but also societal and political ramifications. As per the Epidemiological Unit, Sri Lanka, all cases reported have been traced to a source, confirming the disease being confined to a few clusters (Epidemiological Unit 2020). Nevertheless, with the identification of large clusters from an apparel factory and a fish market within the Western Province, the threat of a transition in to a community transmission stage has become a huge debate in public domain. Given the number and the geographical distribution of primary contacts of aforementioned clusters, it is essential that the health system prepare for a future worst case scenario. The same sentiment has been retreated by the WHO on number of occasions by highlighting that the risk of expanding Covid-19 in to community transmission and countrywide transmission (World Health Organization 2020a). The focus of this paper would be the last two stages namely, the community transmission and the countrywide transmission. For the purposes of this paper, we would amalgamate those two stages to "widespread community transmission". We assumed that during the widespread community transmission, the population of Sri Lanka could be considered as a closed population overtime, since the disease will be spreading homogeneously across communities.

While acknowledging the fact that Sri Lanka has so far being successful in confining the outbreak, as well as having a strong preventive and curative health system, it is still essential to visualize widespread community spread in Sri Lanka would look like (Ministry of Health and Indigenous Medical Services 2017; MSN News 2020; Mukhopadhyay 2020). As per the experience from across the world, widespread community transmission of Covid-19, if not managed properly would be the worst ever public health nightmare. Having an idea of the projected number of cases in the community as well as the numbers who would be hospitalized, those who need intensive care unit treatment and mechanical ventilation may be essential in preparation for a possible community spread or beyond. Visualizing how the community spread of Covid-19 would look like through projections can be a very helpful exercise for healthcare planners, both from preventive and curative sectors. This is especially crucial, since all Covid-19 patients have been offered free care so far, and the government intends to continue to do so. The objective of this 
exercise was to simulate the widespread community transmission of Covid-19 in Sri Lanka, through introducing a single case of Covid-19 to each of the health districts simultaneously.

\section{Methods}

There are various models used to predict the progress of outbreaks. In the current exercise, we used the Susceptibility, Infected and Removed (or recovered) model in predicting the Covid-19 Cases (Li and Zhang 2017; Weisstein 2020b, 2020a). SIR model consists of three compartments:

- Sfor the number of susceptible,

- Ifor the number of infectious, and

- Rfor the number of removed through recovery or death of individuals.

- $\mathrm{N}=\mathrm{S}+\mathrm{I}+\mathrm{R}$,

- $\beta=$ the average number of contacts per person per time

- $\lambda=$ Number of infected individuals

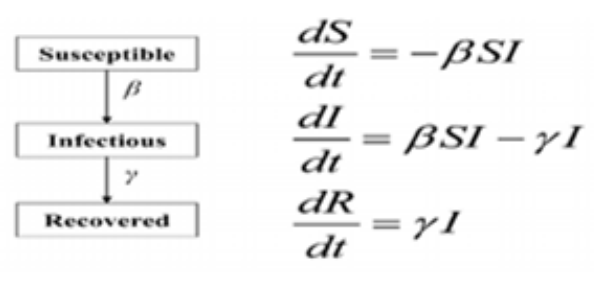

\section{Equation 1 : SIR Model Equation}

SIR model assumes the population to be confined to three compartments, susceptible, infected or recovered. As per the SIR model, an individual would move across different compartments as the outbreak progresses, however, that individual could be present only in one of the three compartments at a given time (Weisstein 2020b, 2020a).

As per Figure 1, the number of susceptible persons will gradually decrease as the number of infected persons gradually rise, peak and decline. Correspondingly, the number of removed persons will gradually increase.

We used COVID-19 Hospital Impact Model for Epidemics (CHIME) Version 1.1.5 developed by Penn State University, which uses a modified SIR model, through the ArcGIS Pro Covid-19 Modelling Toolbox by ESRI (Environmental Systems Research Institute (ESRI) 2020; Weissman et al. 2020). We obtained the Regional Director of Health Services (RDHS) area wise population data from the Department of Census and Statistics through the Medical Statistics Unit of Ministry of Health, Sri Lanka. When deciding the input model parameters, we had two options, either to use inputs based on Sri Lankan data or use the default constant model parameters for the projections. Use of Sri Lankan data as model parameters was 
intuitive. Yet, we found several counter arguments. Firstly, the spread of the disease in Sri Lanka has being confined to specific populations such as returnees to the country, repatriates, Navy sailors, inmates of substance abuse rehabilitation centers, apparel industry employees and fishery community in specific locations, hence information from them model widespread community transmission is irrational and illogical. Secondly, so far, all patients confirmed to be positive for Covid-19 have been hospitalized, which means hospitalization to be $100 \%$, which is challenging to continue during a widespread community transmission. Thirdly, the social distancing going forward to be kept at $45 \%$ as done in the model seems to be quite reasonable from Sri Lankan perspective also, since with the relaxation of the Continous Covid19 curfew, social distancing measures were not promising as it used to be. For these reasons, we decided to adhere to the default constant model parameters used in the USA during the current exercise, as shown in Table 1.

The usual approach in the running of the model is to input the number of currently hospitalized cases to the model, based on the available epidemiological data. Since our objective was to visualize widespread community transmission in Sri Lanka, we thought that modelling in the number of currently hospitalized cases mostly arising from well-defined clusters based on a policy of $100 \%$ hospitalization of Covid-19 patients would be irrational and illogical. Alternatively, we adopted an innovative approach of simulating the widespread community spread of Covid-19 in Sri Lanka by simultaneously introducing one case of Covid-19 to each Regional Director of Health Services area (equivalent to administrative districts) on Day Zero. Correspondingly this would mean having 26 Covid-19 cases on Day Zero of widespread community spread in Sri Lanka. Model was run for a period of 365 days. The outputs were obtained for the country as a whole as well as per RDHS areas.

\section{Results}

SIR model outputs for Sri Lanka for Covid-19 during community spread and beyond in Sri Lanka is shown in Figure 1.

As shown in Figure $1 \mathrm{~A}$, at the time when the first hospitalized case from community spread is reported, the projected total susceptible population will be $20,408,073$ while it would gradually decrease to stabilize at around 10,571,762 at the end of 365 days of projection period. Similarly, the number of persons infected in the community at the time of first admission of the case to hospital would be 1576 persons. The number of infected persons in the community would rise to its peak of 761,932on day 223 of the prediction period, to decreased to 42,137 persons by day 365 . The number of persons removed at the time of the first case being admitted to hospital would be 360 , while this would peak on day 223 to 4776287 and subsequently would reduce to 9796111 by day 365 . 
The daily admissions for Covid-19 during community spread and beyond in Sri Lanka is shown in Figure $1 \mathrm{~B}$.

As per Figure 1B, it is seen that the daily new hospitalizations, daily new ICU admissions and new ventilated admissions will peak to 1942, 583 and 388 by day 215 .

The daily hospital census (Midnight Total) for Covid-19 community spread and beyond in Sri Lanka is shown in Figure 1C.

As per Figure 1C, it is observed that the peak of hospitalized census (midnight total) will be reached on day 218 to 13583. In the meantime, the peak ICU census (midnight total of patients admitted in ICU) and ventilated census (midnight total of ventilated patients) per day will reach on day 219.

As per above map, it is evident that the highest number of hospital census (midnight total) of cases at the peak will be reported from Colombo and Gampaha RDHS areas which will be over 1680 cases per day. Lowest number of hospital census (midnight total) of cases at the peak will be reported from Mannar, Kilinochchi, Mullaitivu and Vavuniya RDHS areas.

\section{Discussion}

Widespread community transmission of the Covid-19 outbreak would be the worst public health nightmare, with rising demands on the health systems with alarming morbidity and mortality (World Health Organization 2020a). Trying to visualize how such a scenario would look like must not be ignored, only reason being the denial of the possibility of such an occurrence. At the outset, it must be noted that predictions are estimates to be used by health systems planners wisely, in the backdrop of many other factors. Unique demographic factors, health seeking behaviours and health system characteristics of a country should be taken in to consideration when applying predictions into actual public health interventions. Further, the methods used to predict the progress of Covid-19 has been quite diverse and debated (Anastassopoulou et al. 2020; Cyranoski 2020; Roda et al. 2020). On the contrary, such drawbacks should not prevent the use of existing methods in visualizing the progress of the outbreak. The results of such predictions should be seen as predictive evidence to formulate public health decisions, than attempts to find faults of health systems and prevailing public health interventions, hence should create a constructive dialogue and a productive debate directed towards pre-emptive, stepwise augmentation of capacity (Weissman et al. 2020).

During the current study, we used an application using the SIR modes, which is one of the simplest compartmental epidemiological models available (Environmental Systems Research Institute (ESRI) 2020; Weissman et al. 2020). The susceptible populations were limited to specific groups since the beginning of Covid-19 in Sri Lanka, starting off with returnees from high risk countries to substance abusers to returning migrants to armed forces (Director General of Health Services 2020). Nevertheless, when the Covid-19 outbreak goes to the community spread, the community will behave more or less in a 
homogeneous way. Hence, the application of the SIR model in homogeneous populations could be justified during widespread community transmission.

During this study, we used a population-based approach in visualizing how the Covid-19 community spread and beyond would look like. Instead of using the past epidemiological data as the basis of the predictions, we simulated the spread of the disease by introducing one case of Covid-19 case at the same time to all RDHS areas. This would be very unlikely in the real-life scenario, however what we wanted to do in this exercise was to have a baseline idea of how widespread community spread would look, if it happens in Sri Lanka. In addition, this was probably the "best" of the worst-case scenarios, since each health districts gets only one case of Covid-19 to start off with, which is, obviously too optimistic to occur in real life. What this implies is that, if the "best" of the worst-case scenarios is alarming, the worse of the worst-case scenarios would be much alarming and demanding. Nevertheless, such a "best" of the worst case scenarios would be more influential in advocating for preparedness, hence all health systems are inherently reluctant to accept that their systems could fail in times of crisis.

According to the predictions, the peak of the outbreak will occur around Day 218 - 220 (after about seven months of the commencing of the community transmission. At the peak, the country may need around 1942, 583 and 388 beds, ICU beds and ventilators per day at the peak. As per the Ministry of Health reports, the total number of hospital beds for treatment purposes in the country as per 30.10 .2020 is to be around 5485 (Director General of Health Services 2020). In the meantime, toal ICU beds in use to be at 146. It is evident seen that even if the community transmission occurs the country may have challenges in catering to the need of beds, ICU beds and ventilators that may arise.

Even though the current policy of Sri Lanka is to admit all patients who are diagnosed with Covid-19 to be admitted to a desingated Covid-19 hospital, this may not continue to be feasible in time of a community transission with increased demand. Hence, it was decided to use the $2.5 \%$ of default value of hospitalization provided in the model, based on statistics from the USA, which is currently going through a community transmission. If the current decision of admitting all Covid-19 patients to a hospital without a triage system, it is likely that the hospitals will be overwhelmed even prior to the peak of the community transmission.

It should be noted that during the current model, artificial demarcation between the stages prior to community transmission and beyond has been made. The number of cases has been reset to zero prior to running the model during the community transmission and beyond. Even though it would be difficult to distinguish between the onset of community transmission and the preceeding stages, for the purpose of the current model, this approach was adopted. In retrospect, the total number of cases during the community transmission and beyond is likely to be much higher than during the preceding stages, hence this approach could be justified.

CHIME model has been added value by incorporating it into the ArcGIS Pro software, hence it was possible to carry out the model for each of the RDHS areas. Even though during the current study, the 
model was run at RDHS level, the same could be done at Medical Officer of Health areas (The grass root level health administrative division) as per the requirement.

However, one possible significant draw back of the CHIME model, as stated out by its developers from the PENN State University was that it recommends only a maximum prediction period of 30 days, however, if the peak does not occur within that period, it is recommended to increase the duration accrodingly(Penn Medicine 2020). It is likely that as one moves further in time, more uncertain the projections become, however, projections still could be useful. We reemphasize the point that we mentioned at the beginning of the discussion on model estimates here. The results of this model should only be treated as estimations, as they are highly sensitive to the model assumptions, data and parameters that we use. Having given consideration for the above facts, during the current study, we used a prediction period of 365 days which was able not only to capture the peak, but alsso the return of the curve to the baseline.

Further research needs to be carried out incorporating factors other than the population such as the actual case number, socioeconomic and demographic vulnerabilities and health systems capacities in predicting widespread community spread in Sri Lanka.

\section{Conclusion}

Widespread community transmission of Covid-19 could be a public health nightmare, Sri Lanka being no exception. It is essential that the health authorities must be ready for the worst-case scenarios, as quantified through this exercise, in order to ensure effective public health response to reduce morbidity and mortality in case of a widespread community transmission.

\section{Declarations}

The authors declare that they have no actual or potential competing interests to declare.

Acknowledgement: GIS Solutions (Pvt) Ltd and the ESRI Disaster Response Program (DRP) for Providing the ArcGIS Pro Software and the technical assistance during the simulation exercise.

This study did not involve human subjects.

\section{References}

Anastassopoulou, Cleo, Lucia Russo, Athanasios Tsakris, and Constantinos Siettos. 2020. "Data-Based Analysis, Modelling and Forecasting of the COVID-19 Outbreak." PLOS ONE 15(3):e0230405.

Cyranoski, David. 2020. "When Will the Coronavirus Outbreak Peak?” Nature.

Director General of Health Services. 2020.“Covid-19 Sitiation Update $=30.06 .2020$," June 30, Colombo, Sri Lanka. 
Environmental Systems Research Institute (ESRI). 2020. ArcGIS Pro. Redlands, CA.: ESRI.

Epidemiological Unit. 2020. Coronavirus Disease 2019 (COVID-19) - Situation Report - 16.07.2020 10.00am. Colombo, Sri Lanka: Epidemiological Unit.

Li, Gui-Hua, and Yong-Xin Zhang. 2017. "Dynamic Behaviors of a Modified SIR Model in Epidemic Diseases Using Nonlinear Incidence and Recovery Rates." PLoS ONE 12(4):e0175789.

Ministry of Health and Indigenous Medical Services. 2017. Annual Health Bulletin - 2017. Colombo, Sri Lanka: Ministry of Health and Indigenous Medical Services.

MSN News. 2020. "Sri Lanka's Success in Countering COVID-19." MSN. Retrieved July 17, 2020 (https://www.msn.com/en-xl/news/other/sri-lanka-s-success-in-countering-covid-19/ar-BB13LIzM).

Mukhopadhyay, Ankita. 2020. "How Sri Lanka Successfully Curtailed the Coronavirus Pandemic." DW.COM. Retrieved July 17, 2020 (https://www.dw.com/en/how-sri-lanka-successfully-curtailed-thecoronavirus-pandemic/a-53484299).

Penn Medicine. 2020. "CHIME App - Model Parameters." Penn Medicine. Retrieved July 17, 2020 (https://code-for-philly.gitbook.io/chime/what-is-chime/parameters).

Roda, Weston C., Marie B. Varughese, Donglin Han, and Michael Y. Li. 2020. "Why Is It Difficult to Accurately Predict the COVID-19 Epidemic?" Infectious Disease Modelling 5:271-81.

Singhania, Meghna A. 2020. "Controversy after Govt Document Uses Limited Community Transmission Word to Describe Current Indian Scenario." Retrieved July 17, 2020

(https://medicaldialogues.in/news/health/government-policies/controversy-after-govt-document-useslimited-community-transmission-word-to-describe-current-indian-scenario-64387).

Weissman, Gary E., Andrew Crane-Droesch, Corey Chivers, ThaiBinh Luong, Asaf Hanish, Michael Z. Levy, Jason Lubken, Michael Becker, Michael E. Draugelis, George L. Anesi, Patrick J. Brennan, Jason D. Christie, C. William Hanson III, Mark E. Mikkelsen, and Scott D. Halpern. 2020. "Locally Informed Simulation to Predict Hospital Capacity Needs During the COVID-19 Pandemic." Annals of Internal Medicine.

Weisstein, Eric W. 2020a. "Kermack-McKendrick Model." Retrieved July 17, 2020

(https://mathworld.wolfram.com/Kermack-McKendrickModel.html).

Weisstein, Eric W. 2020b. “SIR Model." Retrieved July 17, 2020

(https://mathworld.wolfram.com/SIRModel.html).

World Health Organization. 2020a. "Responding to Community Spread of COVID-19: Interim Guidance, 7 March 2020." 
World Health Organization. 2020b. "WHO Coronavirus Disease (COVID-19) Dashboard."

\section{Table}

\section{Table 1 : Constant Model Parameters Used}

\begin{tabular}{|l|l|}
\hline Parameter & Value \\
\hline Doubling time in Days & 5 \\
\hline Social distancing going forward & 45 \\
\hline Date of Social Distancing Measures & Day 1 \\
\hline Hospitalization \% & 2.5 \\
\hline ICU \% (Total Infections) & 0.75 \\
\hline Ventilation \% (Total Infections) & 0.5 \\
\hline Infectious Days & 10 \\
\hline Average Hospital Length of Stay (Days) & 7 \\
\hline Average Days in ICU & 9 \\
\hline Average Days on Ventilator & 10 \\
\hline Source: Environmental Systems Research Institute (ESRI). ArcGIS Pro (version Chime Model v1.1.5). \\
\hline Covid-19 Modelling Toolbox. Redlands, CA.: ESRI, 2020.
\end{tabular}

\section{Figures}




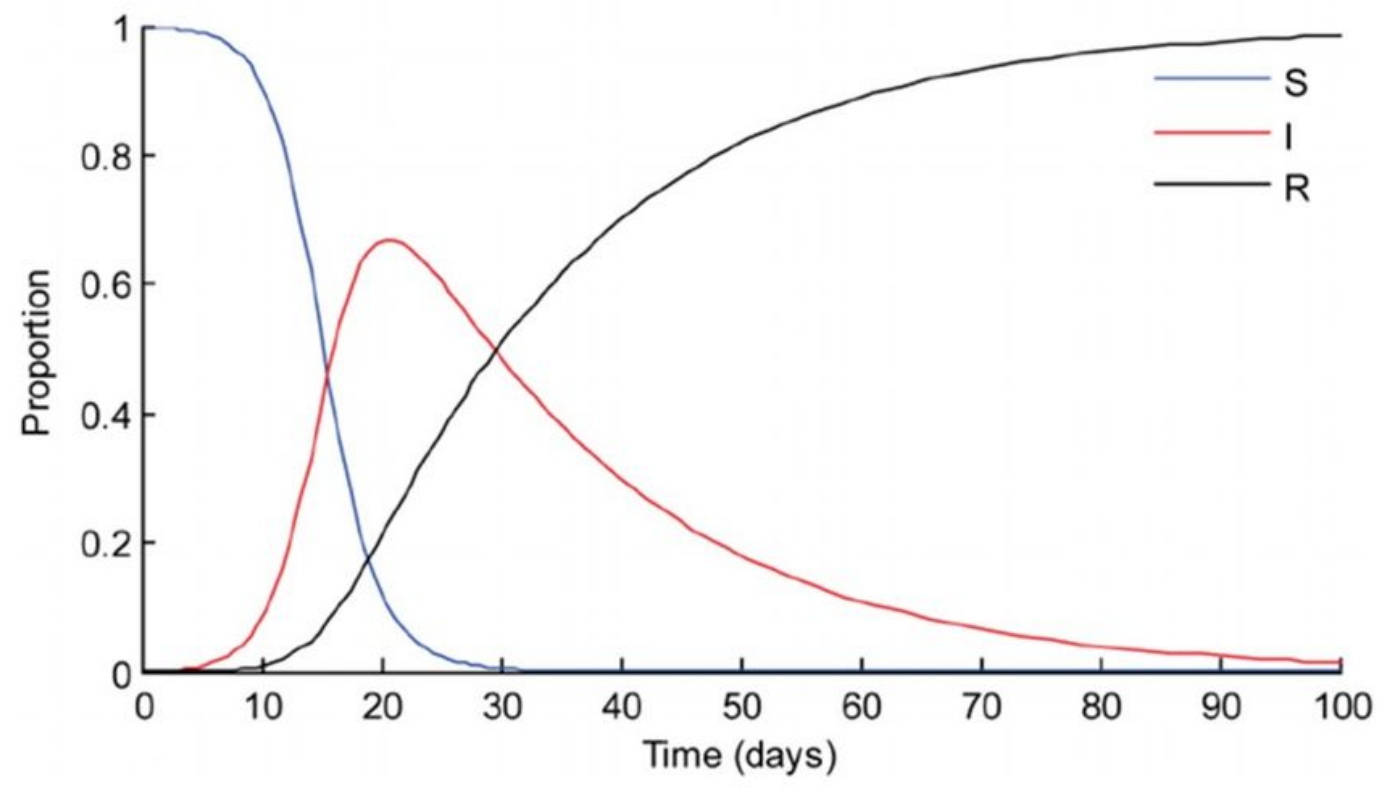

Figure 1: SIR Model

\section{Figure 1}

Susceptible (S), Infected (I) and Removed (R) (SIR) model diagramme shows the progress of fate of the population along the time. The susceptible population gradually decreases, while persons continue to get infected. However, as time passes, the number removed gradually increases as the individuals become recovered, or removed through death. 
A

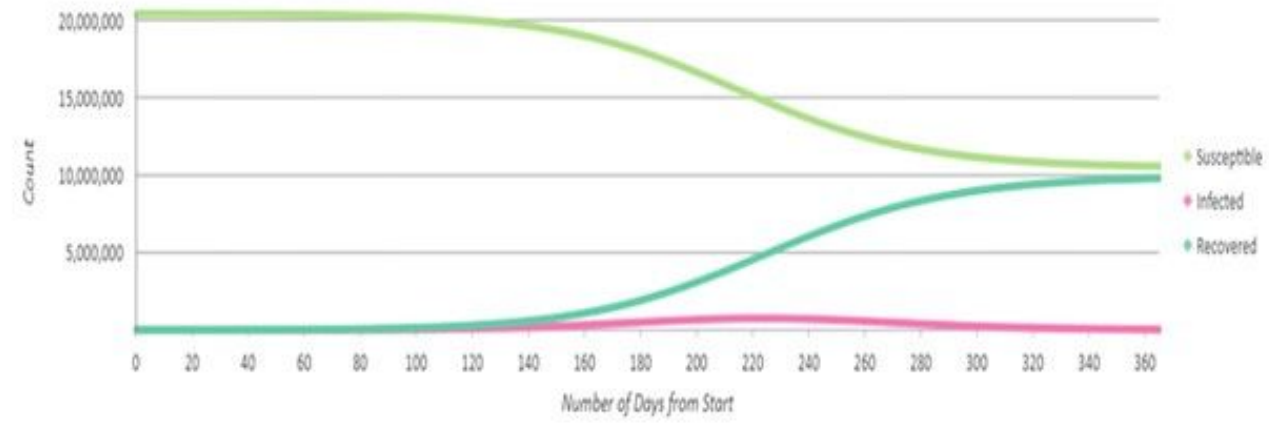

B
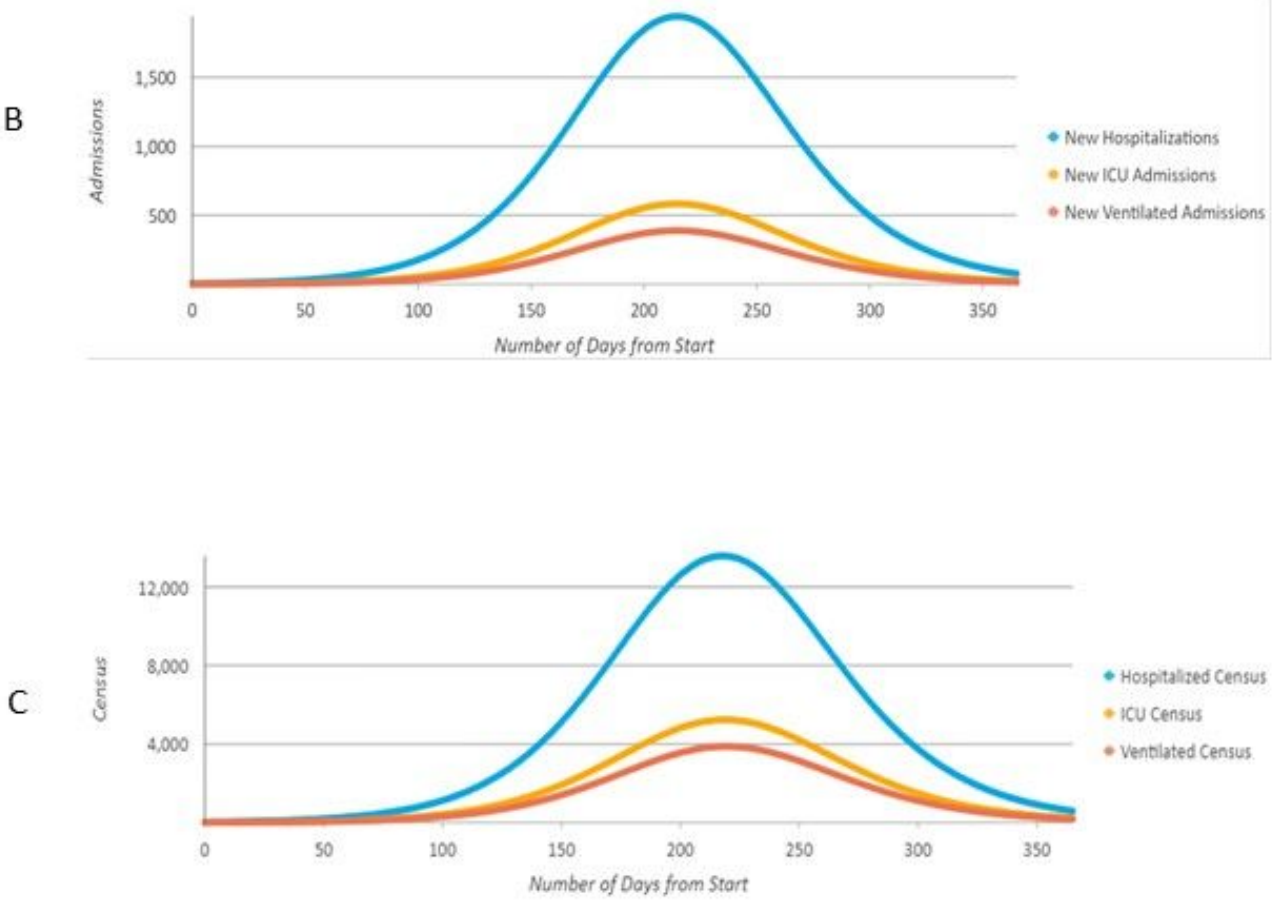

Figure 2 : SIR Model Outputs

\section{Figure 2}

The Susceptible (S), Infected (I), and Removed (R) (SIR) model outputs for the simulated scenario for Sri Lanka are shown. A: This graph shows the relationship between the susceptible, infected, and removed number of patients as per the simulated scenario for Sri Lanka. B: This graph shows the distribution of new hospitalizations, new ICU admissions, and new ventilated admissions per day plotted against the number of days from the start as per the simulated scenario for Sri Lanka. C: This graph shows the 
census (midnight total) of hospitalized patients, ICU patients, and ventilated patients plotted against the number of days from the start as per the simulated scenario for Sri Lanka.

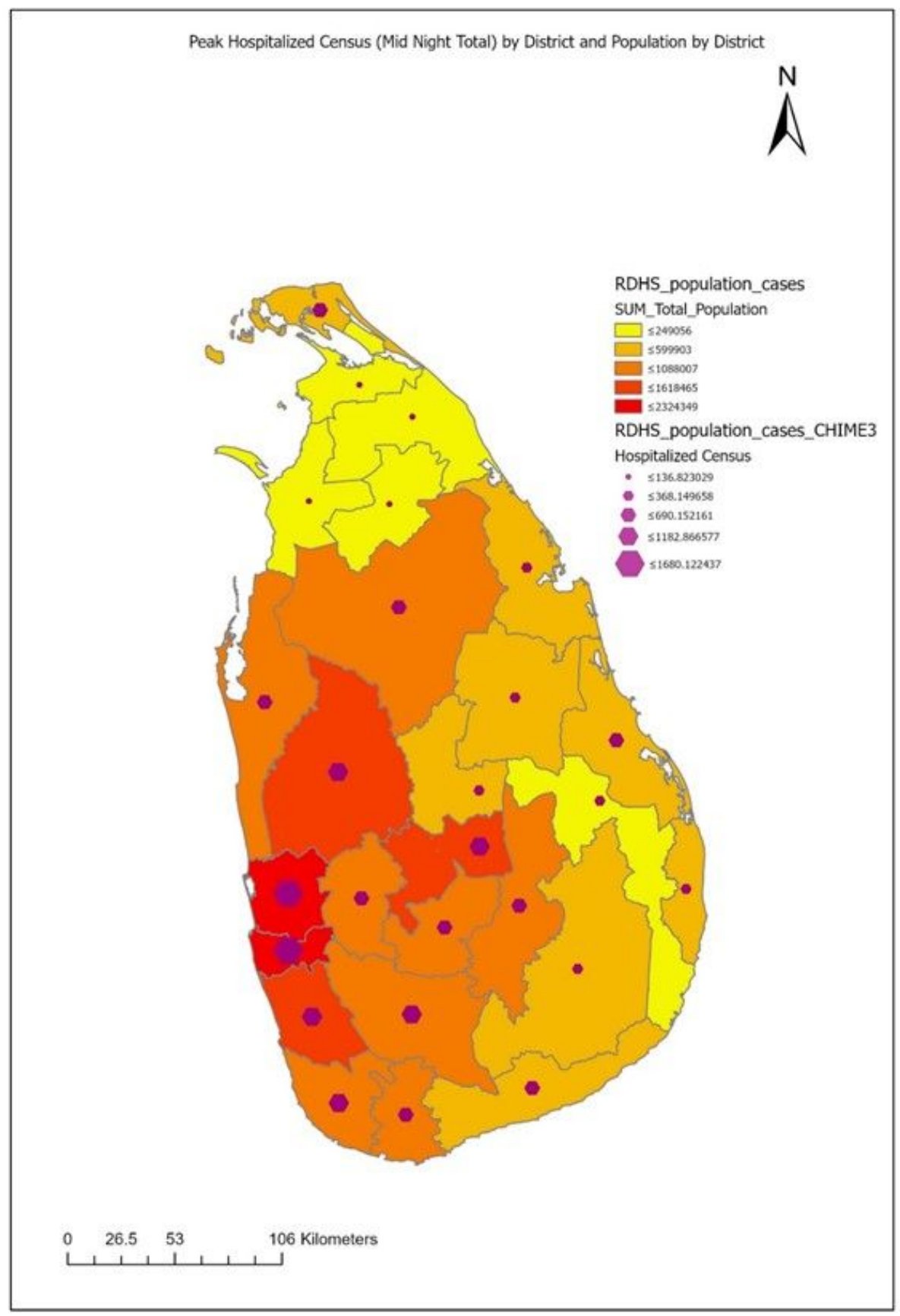

Figure 3 : Peak Hospitalized Census (Mid Night Total) and Population by District

\section{Figure 3}

This map shows the hospitalized census (midnight total) at the peak of the outbreak. The graduated symbols show the distribution of numbers at the peak. The background map shows the distribution of the population by the district. 